Décadrages Décadrages

cinéma, à travers champs Cinéma, à travers champs

$31 \mid 2015$

Education au cinéma

\title{
Quand les mutations des pratiques audiovisuelles des jeunes réveillent les enjeux de l'éducation aux médias
}

Marlène Loicq

\section{(2) OpenEdition}

Journals

Édition électronique

URL : https://journals.openedition.org/decadrages/827

DOI : $10.4000 /$ decadrages. 827

ISSN : 2297-5977

Éditeur

Association Décadrages

Édition imprimée

Date de publication : 1 octobre 2015

Pagination : $35-45$

ISBN : 9782970096313

ISSN : 2235-7823

Référence électronique

Marlène Loicq, «Quand les mutations des pratiques audiovisuelles des jeunes réveillent les enjeux de l'éducation aux médias », Décadrages [En ligne], 31 | 2015, mis en ligne le 29 mai 2018, consulté le 26 mars 2023. URL : http://journals.openedition.org/decadrages/827 ; DOI : https://doi.org/10.4000/ decadrages. 827

Creative Commons - Attribution - Pas d'Utilisation Commerciale - Partage dans les Mêmes Conditions 4.0 International - CC BY-NC-SA 4.0

https://creativecommons.org/licenses/by-nc-sa/4.0/ 


\section{Quand les mutations des pratiques audiovisuelles des jeunes réveillent les enjeux de l'éducation aux médias}

L'ÉDUCATION AUX MÉDIAS EST un domaine balisé, fort d'une histoire et de fondements théoriques inscrits dans les sciences de l'information et de la communication (SIC), qui en font un projet de plus en plus intégré aux curriculums scolaires de nombreux pays. Si les formes institutionnelles qu'elle prend diffèrent d'un contexte à un autre ${ }^{1}$, c'est à la fois pour répondre à des politiques éducatives distinctes et parce que le projet même d'éduquer aux médias se présente en réponse à des enjeux socio-éducatifs déterminés en partie par la façon dont on appréhende la relation des jeunes aux médias. Ceux-ci sont parfois considérés comme manipulateurs, dangereux pour les jeunes, ou au contraire, comme porteurs d'un fort potentiel éducatif et créatif. Selon la démarche éducative envisagée, les médias sont utilisés comme outil pédagogique (éducation par les médias) ou analysés dans leurs contenus et fonctionnement (éducation aux médias). La perspective adoptée fera alors appel à des appareillages théorique et méthodologique distincts desquels se dégagent quelques approches largement partagées ${ }^{2}$. Qu'elles soient critiques, réflexives, normatives ou sémiologiques, ces approches plongent leurs racines dans les théories de la communication de masse: de la théorie des effets ${ }^{3}$ aux approches critiques (voir l'Ecole de Francfort, I930I960); des effets limités à la perspective des usages ${ }^{4}$; et de la perspective sémiologique ${ }^{5}$ aux Cultural Studies (voir l’Université de Birmingham) et aux médiacultures ${ }^{6}$. L'éducation aux médias s'est donc construite

1 Marlène Loicq, «Médias et Interculturalité: l'éducation aux médias dans une perspective comparative internationale (Australie, Québec, France)», thèse de doctorat en cotutelle Université Paris III Sorbonne Nouvelle, Université Laval, Québec, 20II; Jacques Piette, L'Educa- tion aux médias et fonction critique, Montréal, L'Harmattan, 1996.

2 Jacques Piette, La Filiation théorique entre les programmes d'éducation aux médias et les courants de la recherche sur la communication de masse, mémoire de maîtrise, Université de Sherbrooke,
I988; James A. Anderson, «Television literacy and the critical viewer», dans Bryant J., Anderson D.R. (éd.), Children's Understanding of Television: Research on Attention and Comprehension, San Diego, Academic Press, I983; Len Masterman, Teaching the Media, Londres, Routledge, I985.

3 Harold D. Lasswell, Propaganda Technique in the World War, New York, éditions Knopf, 1927.

4 Elihu Katz, Paul Lazarsfeld, Personal Influence: The Part Played by People in the Flow of Mass Communications, New York, Free Press, i955.

5 Roland Barthes, Mythologies, Paris, Seuil, I957; Christian Metz, Essais sur la signification au cinéma, tome 2, Paris, Klincksieck, I972; Christian Metz, «Images et pédagogie», Communications, $\mathrm{n}^{\circ}$ I5, 1975 .

6 Eric Maigret, Eric Macé, (éd.) Penser les médiacultures, Nouvelles pratiques, et nouvelles approches de la représentation du monde, Paris, Armand Colin/INA, 2005; Geneviève JacquinotDelaunay, «De l'éducation aux médias aux médiacultures: faire évoluer théories et pratiques", Ina Sup, Pôle européen des sciences et métiers de l'image et du son, 20I0 www.ina-sup.com/node/I579; Henry Jenkins, Convergence Culture: Where Old and New Media Collide, New York, New York University Press, 2006. 
7 Ces deux définitions sont disponibles sur le site Eduscol.education.fr.

8 Geneviève Jacquinot-Delaunay, "Media education: when the waiting is over...» dans Ulla Carlsson, Samy Tayie, Geneviève Jacquinot-Delaunay, José Manuel Pérez Tornero (éd.), Empowerment through Media Education, An intercultural Dialogue, Göteborg, ICCYM, UNESCO, Dar Graphit, Mentor Association, 2008. corrélativement aux recherches sur les médias, dans une démarche à la fois éthique (autour d'enjeux citoyens notamment) et pratique (autour de la philosophie du «learning by doing»).

Ces enjeux éducatifs et sociaux qui ont conduit à la mise en place de projets éducatifs autour des médias puis à l'institutionnalisation de l'éducation aux médias comme domaine se transforment en même temps qu'évoluent les médias eux-mêmes. Aujourd'hui, pour la commission européenne, l'éducation aux médias c'est la «capacité à accéder aux médias, à comprendre et apprécier, avec un sens critique, les différents aspects des médias et de leur contenu et à communiquer dans divers contextes». L'inspection générale française la définit ainsi: «Toute démarche visant à permettre à l'élève de connaître, de lire, de comprendre et d'apprécier les représentations et les messages issus de différents types de médias auxquels il est quotidiennement confronté, de s'y orienter et d'utiliser de manière pertinente, critique et réfléchie ces grands supports de diffusion et les contenus qu'ils véhiculent. ${ }^{7}$

Si le cœur du projet reste de fournir des outils critiques pour comprendre et utiliser les médias, de développer des compétences liées à la culture médiatique, l'apparition de chaque nouveau média et la transformation associée des contenus impose de repenser les moyens et finalités de ce projet. En effet si les diverses approches éducatives autour des médias sont intimement liées à leur «importance» dans le social ${ }^{8}$, celle-ci est en constante mutation. Dans les années i960 et 70, les films étaient mobilisés dans une éducation à l'image essentiellement esthétique et linguistique. Ce sont ensuite la télévision et les approches critiques qui ont dominé les années i980. L'apparition de nouvelles formes de communication de masse a confirmé l'importance de cette approche autour des questions du rapport local/global posé notamment par l'arrivée de la vidéo personnelle et la naissance des chaînes privées. Internet et le Web dans les années I990 ont à nouveau modifié l'approche éducative des médias, l'orientant plus sur des compétences techniques. Aujourd'hui, la numérisation des contenus et la diversification des services culturels offerts par les différents médias rendent confuse la distinction par support médiatique tant ils se retrouvent et se rencontrent au sein des industries culturelles. Alors quels sont les nouveaux enjeux liés à cette dernière évolution médiatique?

La convergence des langages et la «multimédiatisation» doivent conduire à une éducation aux médias de plus en plus complexe qui, 
comme toujours, s'appuie d'abord sur les pratiques médiatiques des jeunes. Pour cela, la première étape est donc bien évidemment de les connaître et de les comprendre. Ce texte propose alors de repenser les enjeux de l'éducation aux médias à l'heure du numérique à partir d'une enquête sur les pratiques télévisuelles et numériques des jeunes ${ }^{9}$. Il s'agit dans un premier temps de rappeler le cheminement institutionnel des éducations aux médias et au cinéma pour ensuite, dans un deuxième temps, présenter quelques résultats de ladite enquête en vue de comprendre les permanences et mutations de la posture spectatorielle des jeunes, et enfin de conclure sur ces nouveaux enjeux à prendre en compte pour penser l'éducation aux médias aujourd'hui.

\section{L'éducation aux médias en réponse aux pratiques audiovisuelles des jeunes}

L'éducation aux médias est apparue quasi simultanément aux médias eux-mêmes et a toujours suivi leur progression dans la vie sociale. Ce projet éducatif doit être initié par les pratiques médiatiques elles-mêmes et répondre ainsi à des enjeux concrets dans le rapport des jeunes aux médias et plus largement au monde.

Si l'approche française de l'éducation aux médias est largement ancrée dans une distinction par support médiatique, la presse y occupe beaucoup de place, au détriment de l'audiovisuel. Pourtant, le cinéma a su trouver un espace de développement éducatif grâce notamment à son statut artistique qui lui confère une légitimité éducative dans le domaine des arts (alors que parallèlement, l'éducation aux médias est plutôt associée à une démarche citoyenne). Nous déplorons bien sur cette distinction qui prive le cinéma de sa profondeur signifiante et ne permet pas, comme le disent Boillat et Graff ${ }^{10}$, d'« apprendre à poser un regard sur le film en se demandant comment celui-ci pose un regard sur le monde». Et nous le déplorons pour l'éducation aux médias qui renvoie une image erronée en France car, si l'enjeu citoyen est grand, il ne passe pas seulement par la presse et la défense du pluralisme, mais aussi par une éducation à la fiction, format préféré et structurant des jeunes dans leur rapport au monde.

Ceci étant dit, il est primordial de se rappeler qu'au-delà des déclarations officielles, c'est d'abord sur le terrain que l'éducation aux médias existe, prônée et enseignée par des éducateurs engagés. Bien avant l'institutionnalisation du domaine, des initiatives d'éducation aux
9 Projet «Jeunes, numérique et télévision» (JNT), 20ı4, dirigé par Amandine Kervella, Elodie Kredens, Marlène Loicq et Florence Rio, financé par une BQR de Lille Métropole et la Région Rhône-Alpes via Fréquence Ecole.

10 Alain Boillat, Séverine Graff, "L'étude du cinéma, de l’Université de Lausanne aux classes du Secondaire», Jeunes \& Médias. Les cahiers francophones de l'éducation aux médias, $\mathrm{n}^{\circ}$ 7, 2015, np. 
11 Il est cependant impossible de le faire dans le cadre de cet article; sur ce point, voir Divina Frau-Meigs, Marlène Loicq, Perrine Boutin, «Politiques d'éducation aux médias et à l'information en France», Rapport remis à l'UNESCO et le Conseil de l'Europe dans le cadre du projet Media and Information Literacy Policies in Europe, 20I4; Marlène Loicq (coord.), «L'éducation aux médias dans le monde», Jeunes \& Médias, les cahiers francophones de l'éducation aux médias, $\mathrm{n}^{\circ}$, Paris: Publibook Université (EPU), collection revues, 2013; Len Masteman, François Mariet, L'Education aux médias dans l'Europe des années 80, Strasbourg, Editions du Conseil de l'Europe, I994.

12 Marlène Loicq, "Les discours officiels sur les médias dans l'éducation: de la théorie à la politique. Etude comparative internationale», dans Béatrice Vacher, Christian Le Möenne, Alain Kiyindou (éd.), Communication et débats publics: les réseaux numériques au service de la démocratie?, Paris, L'Harmattan, collection communication et civilisation, 2013.

13 Christophe Gauthier, La Passion du cinéma, op. cit., I999, p. 68. médias sont apparues dans divers pays et différents contextes, de manière quasi simultanée et leur histoire mériterait que l'on s'y attarde "1. Leur officialisation s'est ensuite faite selon des temporalités propres et des logiques associées au fonctionnement même des systèmes scolaires concernés ${ }^{12}$.

En France, le cinéma se constitue dès 1984 en enseignement optionnel dans les classes de $2^{\text {nde }}$ et continue son rapprochement avec le milieu éducatif amorcé dès 1908 avec le Rapport d'Edmond Benoît-Levy sur L'Application du cinéma à l'instruction. L'éducation aux médias doit attendre la loi d'orientation et de programmes pour l'avenir de l'école adoptée par le Ministère de l'éducation nationale, de l'enseignement supérieur et de la recherche (MENESR) en 2005 pour apparaître officiellement dans les programmes scolaires. Pourtant, l'instance qui lui est dédiée (le Centre de liaison de l'enseignement et des médias d'information - CLEMI) travaille activement à son intégration et à sa reconnaissance depuis le début des années i980.

Le rapprochement de l'école et du cinéma a connu des étapes intermédiaires d'institutionnalisation. En I9II, le ministère de l'Instruction publique crée le Service du cinématographe à l'école et il en confie le fonctionnement au Service des vues fixes du Musée pédagogique. Se tient en 1922 le $\mathrm{I}^{\text {er }}$ Congrès national du cinéma éducatif à l'initiative de la Direction générale de l'enseignement technique. Il y est développé l’idée qu'il ne faut pas instrumentaliser le cinéma à l'école sans prendre en compte les qualités artistiques inhérentes aux films. Ce congrès a pour but d'affermir l'essor de l'enseignement artistique et technique en milieu scolaire, et l'une des revendications est d'utiliser le «cinéma dans toutes les écoles ${ }^{13}$.

Ce rapprochement a aussi connu des initiatives associatives et civiles avec notamment la création des «ciné-clubs» et des associations telles que les Amis du cinéma. La vocation pédagogique et la volonté d'apprendre à lire les images y sont prédominantes, le tout assorti d'une conviction cinéphile permettant de dépasser la simple instrumentalisation du cinéma dans un but éducatif. Des instances se mettent en place autour des offices régionaux du cinéma éducateur, l’UFOLEIS (Union française des œuvres laïques d'éducation par l'image et par le son), l'ANPEDU (Association nationale pour la promotion des arts de l'écran dans l'université), le Centre national de documentation pédagogique 
(CNDP). La constitution de la COSEAC (Commission d'orientation et de suivi des enseignements et activités de cinéma-audiovisuel) en 1990 est suivie en 1992 par la création des PLEA (Plans locaux d'éducation artistique) incluant le cinéma et l'audiovisuel. A partir de I993, se mettent en place divers dispositifs «Ecole et cinéma» (primaire), "Collège au cinéma» (secondaire), «Lycéens et apprentis au cinéma» (lycée) ainsi que des rencontres pour l'éducation au cinéma et à l'audiovisuel en 1999. Pour l’année 20ı-20II, les trois dispositifs ont mobilisé I 406973 élèves, 50000 enseignants, plus de 2000 salles de cinéma et ont généré 3,7 millions d'entrées en salles ${ }^{14}$. Il apparaît que l'éducation au cinéma a trouvé une place dans l'éducation artistique et culturelle de l'institution. Pour les autres écrans, la situation est plus difficile car ils sont victimes de la dichotomie française entre culture haut de gamme (le cinéma comme $7^{\mathrm{e}}$ art) et la culture bas de gamme (télévision, jeux, bande dessinée).

L'éducation aux médias a quant à elle sauté les étapes institutionnelles car entre la lettre de reconnaissance du rapprochement de la presse et de l'école du ministre de l'Education René Haby en 1976 et la création du Socle commun de compétences et de connaissances par le MENESR en 2006, ce sont surtout les initiatives associatives qui portent le projet. C'est aussi dans des démarches interministérielles qu'il se dessine car si ces nouvelles approches trouvent une place importante dans l'éducation populaire, les modifications des éducations formelles dans le milieu scolaire restent plus minoritaires, jusqu'à la mise en place de projets académiques et nationaux qui, grâce à leur ampleur, laisseront des traces plus sensibles sur le système éducatif. Il s'agit des projets de l'Institut coopératif de l'école moderne (ICEM), porté par la pédagogie Freinet et du cours d'Initiation à la communication audio-visuelle (ICAV) développé par René La Borderie en 1965. En I97I, l'ICAV devient l’ICOM (Initiation à la communication et aux médias), ouvrant alors à l'intégration plus large des questions de communication, et donc, se détachant du support pour se tourner vers l'éducation aux médias. Ce projet et les évaluations qui en découlent donnent lieu à des recommandations quant à l'intégration de l'audiovisuel à l'école, ce qui est renforcé par le rapport Nora/Minc en 1978 sur l'informatisation de la société.

Parallèlement à cela, et ouvrant sur des préoccupations audiovisuelles étendues à d'autres médias que le cinéma, des initiatives interministérielles voient le jour avec la création d'associations telles que le
14 Centre national du cinéma, juillet 2013, Rapport d'activité 2012 du CNC, p. 3 I. 
15 Alain Boillat, Séverine Graff, op. cit., np.

16 Amandine Kervella, Marlène Loicq, «Les pratiques télévisuelles des jeunes à l'heure du numérique: entre mutations et permanences», Etudes de Communication, Dossier thématique $\mathrm{n}^{\circ} 44,2015$.
JTA (Jeunes téléspectateurs actifs). En partenariat avec l'INA (Institut national de l'audiovisuel), certaines chaînes de télévision et divers ministères, tentent des initiatives de rapprochement des mondes de l'école, de la famille, du milieu socio-culturel, etc. Les conséquences de ce projet se trouvent essentiellement dans les instructions officielles de 1985 pour les écoles et les collèges qui retiendront l'idée d'une éducation aux médias citoyenne et critique.

\section{Quand les pratiques médiatiques changent...}

Si le rapport aux images a changé de par la posture même du spectateur, sa capacité d'accès à une palette de plus en plus large de formats, genres, dispositifs et statuts d'images diverses pose de nombreuses questions aux éducateurs, qu'ils soient dans une démarche d'éducation aux médias ou plus spécifiquement au cinéma car «contrairement à ce que pourrait laisser croire le discours majoritairement esthétique des cinéphiles, l'étude du cinéma, lorsque ce dernier est envisagé plus largement comme dispositif et dans la richesse de ses généalogies et filiations, est centrale pour appréhender les «médias, images et technologies de l'information $\gg{ }^{15}$.

Ce rapport à l'image et à l'expérience audiovisuelle s'est évidemment modifié avec l'arrivée de la télévision, puis de la vidéo personnelle et enfin avec internet et un accès accru à toutes sortes de contenus vidéo et des postures spectatorielles modifiées ${ }^{16}$. La posture d'écoute et le contrat de communication dans une salle de cinéma sont basés sur la volonté, la sélection, la linéarité et une temporalité très contrainte (horaires du film programmé et (non) pauses imposées, avec obligation de quitter la salle une fois le film terminé). La posture spectatorielle télévisuelle est plus participative puisque le téléspectateur a le choix du film (selon une grille imposée mais avec une offre plus large que les salles de cinéma, et surtout, télécommande en main, il a le choix de «zapper» en cours de visionnage). Le téléspectateur peut aussi avoir un degré d'attention moindre puisque la situation de la télévision au cour du foyer lui permet de s'adonner simultanément à de multiples tâches (le visionnage télévisuel étant ou non l'activité principale). La numérisation des contenus audiovisuels et l'arrivée d'outils de communication sophistiqués (associés à internet, tels que les Box, les services de $V O D$, mais aussi les sites des chaînes avec offre de replay par exemple, ou les plateformes 
mêlant tous types de contenus audiovisuels) ont la possibilité de modifier très profondément les postures spectatorielles. En effet, le visionneur n'est plus contraint temporellement (par exemple, il peut choisir à quel moment il regarde un film, indépendamment de la grille de programme ou de la sélection de son cinéma; à quel rythme, puisqu'il peut accélérer, rediffuser ou même sauter certains passages; dans une temporalité qui n'est plus seulement imposée par les industries culturelles concernées - le chaînon sortie cinéma, puis DVD puis télévision payante puis hertzien n'est plus respecté; de même qu'il peut regarder une sortie américaine, en France, quasi simultanément en bénéficiant des services de sous-titrages amateurs - voir phénomène «fansub»). Finalement, le consommateur de contenus audiovisuels peut dorénavant composer son visionnage à peu près comme il le souhaite.

Co-existent alors des postures spectatorielles dites «classiques», associées à une situation particulière de visionnage et des postures que l'on peut qualifier d'«émergentes», plus autonomes et diversifiées car les conditions sont choisies par le spectateur lui-même. Du côté des médias, cette ambivalence est entretenue entre la persistance d'une offre historiquement liée à chaque support, et le développement parallèle de nouveaux dispositifs plutôt transmédias. Comme le souligne François Jost ${ }^{17}$ :

«désormais, chacun des médias réutilise l'autre dans un mouvement d'autoréflexion et d'hybridation lié à une circulation intense entre écrans divers, ceux des salles, des musées, de la télévision, des ordinateurs. Le cinéma se trouve donc englobé dans une véritable nébuleuse médiatique, tout en maintenant encore son statut privilégié grâce à des spectateurs inconditionnels».

S’il est aisé d’identifier les changements proposés par les industries culturelles, qu'en est-il des pratiques? Que font les jeunes de ces nouveaux dispositifs? Ont-ils profondément modifié leurs pratiques audiovisuelles? Je répondrai en partie à ces questions à partir des résultats d'une large enquête menée auprès de 2600 jeunes des régions françaises RhônesAlpes et Nord, âgés de 12 à 25 ans. Cette étude menée en 20I4, nommée «Jeunes, Numérique et Télévision», interroge précisément les mutations et permanences des pratiques télévisuelles à l'heure du numérique.

Le secteur de la télévision se mobilise pour renouveler ses dispositifs et proposer une expérience télévisuelle plus complète (appel au transmédia),
17 François Jost (éd.), Comprendre les médias, Paris, Edition Bréal, 2009, p. 85. 
18 Jean-Samuel Beuscart, Thomas Beauvisage, Sisley Maillard, «La fin de la télévision? Recomposition et synchronisation des audiences de la télévision de rattrapage», Réseaux, ${ }^{\circ}{ }^{175}$, 2012, pp. $43-82$.

19 Dominique Boullier, La Télévision telle qu'on la parle. Trois études ethnométhodologiques, L'Harmattan, coll. Champs visuels, Paris, 2003; Sylvie Octobre, Christine Detrez, Pierre Mercklé, Nathalie Berthomier, L'Enfance des loisirs, Paris, DEPS, 2010; Dominique Pasquier, Cultures lycéennes. La tyrannie de la majorité, Paris, Autrement, 2005.

20 François Jost, «Où va la télévision? De l'influence de la télévision numérique sur les programmes et la programmation», dans Gilles Delavaud (éd.), Permanences de la télévision, Paris, Editions Apogée, 20II, p. 30.

21 Dominique Cardon, La Démocratie Internet. Promesses et limites, Paris, Seuil, coll. «La République des idées», 20 Io. plus participative (appel à la social TV), et plus créative (appel à la coproduction). Elle tente de muter.

Face à cela, il apparaît en premier lieu que la posture spectatorielle des jeunes présente une forte propension à la permanence, avec une consommation télévisuelle qui reste dans le flux, sur l'écran de télévision et assez peu participative. Elle conserve une forte logique sociale avec son rôle traditionnel de suggestion de contenus ${ }^{18}$, mais aussi d'agrégateur social en étant un sujet de discussion, de rendez-vous ${ }^{19}$, et, phénomène plus récent, de partage (permis grâce à une dématérialisation des contenus).

Parallèlement, on voit aussi apparaître une forme d'appropriation de certains des dispositifs permettant notamment une consommation plus «à la carte» avec l'usage du replay et de l'extrait, notamment sur des plateformes telles que Youtube. Le rapport à l'offre numérique des chaînes les plus regardées par les jeunes est quant à lui assez limité. Ce constat est notamment visible au regard des taux de fréquentation $(72 \%$ des répondants ne visitent pas les sites des chaînes), des différents usages (lorsqu'ils vont sur ces plateformes, c'est pour visionner des replays: 76,2\%; avoir des informations: $53,8 \%$; voir des extraits: $32,5 \%$; voir des vidéos bonus : $28,4 \%$ ) et selon les types de participation (4,3\% disent participer à des forums, 6,1\% discuter avec des membres, et ı, $2 \%$ écrire des commentaires).

Or, même si «à chaque fois que la télévision sait créer un événement dans le flux et le relayer par internet, elle ramène des jeunes à elle ${ }^{20}$, il semblerait que les dispositifs numériques associés aux émissions et programmes préférés des jeunes ne rencontrent pas l'engouement attendu, et la participation (et a fortiori la production) restent des pratiques assez marginales comme cela a pu être montré par ailleurs ${ }^{21}$. Pourtant, en 2013 et pour la première fois, les $15-24$ ans passaient plus de temps devant leur écran d'ordinateur que devant celui de la télévision (IIh5o/semaine pour la télévision contre I3h3o pour l'ordinateur, Ipsos 2013). Avec l'apparition d'internet, une forte concurrence audiovisuelle est apparue, non seulement dans les logiques de diffusion (plus ou moins légales) mais aussi de production (de plus en plus de jeunes plébiscitent voire produisent des contenus amateurs, notamment dans le domaine sportif et humoristique, et créent un marché audiovisuel parallèle qui tend de plus en plus à emprunter les codes des industries culturelles sans en 
faire totalement partie). Ces "pro-am» (terme issu d'une tension lexicale née de «discours de représentation antagonistes» ${ }^{22}$ ) sont souvent observés comme des terrains d'expérimentation socio-économique qui se mettent en œuvre hors des contraintes et capacités des industries culturelles, tout en étant pour certains, source de bénéfices. Cet utilisateur dont on prône l'empowerment sans vérifier ses véritables désirs et possibilités de se saisir de ces dispositifs liés à la participation et à la production audiovisuelles est-il averti de sa participation à un marché de l'audiovisuel? Est-il outillé pour s'emparer de manière créative et raisonnable de ces dispositifs? Est-il finalement éduqué à cette nouvelle donne audiovisuelle?

\section{De nouveaux enjeux pour une éducation aux médias toujours en chantier}

Entre relative permanence d'une posture et profondes mutations d'une offre, les enjeux de l'éducation aux médias doivent être repensés. Plus que jamais la circulation entre trois pôles que sont: les réceptions, les produits et les productions, sont à articuler, confronter, agencer. Ce travail peut se faire à partir de plusieurs questionnements. Celui des «effets» ${ }^{23}$ est le plus courant et le plus simple. Il s'agit d'interroger des «effets visés» par les logiques de production du côté de l'industrie culturelle; des « effets produits » par une analyse sémio-pragmatique de contenus; et des «effets réels » par une approche du côté de la réception qui doit se faire de manière individuelle mais aussi collective, la réception étant elle-même issue d'une pratique sociale. Le plus important dans cet exercice est bien sûr de ne pas dissocier ces différents moments, ces «effets», d'un contexte particulier, et de ne pas les désarticuler puisqu'ils sont intimement corrélés.

Lélément central dans cette éducation aux médias à trois pôles est celui de la question du sens. Au niveau de la production, on s’interrogera sur les intentions signifiantes (et stratégiques en intégrant une dimension critique) dans la création, la production et la diffusion de certains contenus; la question du sens sera évidemment centrale dans l'étude des contenus audiovisuels eux-mêmes notamment avec l'étude des représentations («que dit-on du monde?»); et l'approche par la réception sera focalisée sur les mécanismes d'interprétations à la fois dans les capacités à redonner du sens (individuellement et collectivement), et dans les impacts que cela a sur le rapport au monde.
22 Guy Lochard, «L'appel aux amateurs dans la $\langle$ TV2.0 $\rangle$, Des discours de représentation aux produits attestés» dans Gérard Leblanc, Sylvie Thouard (éd.), Numérique et Transesthétique, Villeneuve d'Asq, Presses Universitaires du Septentrion, Collection «Arts du Spectacle-Images et sons», 2012.

23 Il ne s'agit ici évidemment pas de la théorie des effets (Harold D. Lasswell, Propaganda Technique in the World War, New York, Knopf, 1927) mais bien du triptyque sémio-pragmatique «production - dispositif - réception» (Patrick Charaudeau (éd.), La presse, Produit, Production, Réception, Paris, Didier Erudition, I988) qui permet dans ce cadre éducatif d'appréhender l'effet comme référence et comme réaction, dans une approche à la fois sémiologique, sociologique et psycho-cognitive. 
D’autres questionnements sont évidemment possibles dans cette éducation aux médias qui doit absolument intégrer les trois pôles mentionnés dans une approche associée, conjointe, articulée, car la convergence numérique conduit à des mécanismes complexes qui structurent dorénavant le secteur audiovisuel. L'enjeu est de taille puisqu'il s'agit en fait de simplifier la compréhension des médias à mesure que leurs fonctionnements se complexifient.

Si l'éducation à l'image n'a pas seulement une fonction esthétique ou citoyenne comme nous venons de le voir, cette nouvelle posture ne fait que renforcer les enjeux liés à la compréhension des divers mécanismes de production et de circulation des contenus produits par les industries culturelles, de même que ceux produits hors de ces circuits.

En effet, du point de vue esthétique, l'expansion des modèles et contenus audiovisuels a bien sûr fait apparaître une multitude de genres nouveaux, de codes et d'esthétiques qui sont souvent des métissages, des rencontres et imposent un regard nouveau sur l'idée même de mise en scène. De plus, la multiplication et la permutation des supports ont fait naître un phénomène de transmédiation qui conduit à se pencher plus sérieusement encore sur la notion de narration. Or ces thématiques, fondamentales en éducation aux médias, doivent être repensées à l'aune de ces nouveaux dispositifs et des usages qui en sont faits. Enfin, c'est bien l'approche des industries culturelles qui doit être également remaniée tant celles-ci sont bouleversées par les mutations en cours de leurs différents secteurs.

L'éducation aux médias sera toujours en chantier. L'évolution rapide des NTIC et des pratiques sociales qui y sont associées rendent ce domaine d'autant plus complexe. Cette mutation perpétuelle et la nécessité d'associer le projet pédagogique qui l'accompagne à l'individualité des pratiques expliquent la difficulté qu'a l'école à lui faire une place pérenne. Si les éléments de compréhension des industries culturelles, des codes sémiotiques et des mécanismes de réception sont des bases stables à une éducation aux médias, il faut veiller à ne pas les séparer, et surtout, à ne pas les déconnecter des pratiques des jeunes pour qu'elle reste pertinente.

Par ailleurs, il est impératif d'être vigilant face aux diverses représentations qui constituent en partie notre rapport à ces questions. Pour l'adulte, les jeunes sont trop souvent des «natifs» pour qui les NTIC n'ont 
pas de secret dans leur usage, mais envers lesquels ils ne savent être critiques. Or c'est là précisément passer à côté des réels besoins éducatifs de ces jeunes qui, comme le montre l'enquête JNT, ne se saisissent pas massivement des dispositifs offerts. Cette permanence dans une posture spectatorielle dite «classique» est autant de sécurité pour l'éducateur qui avance en terrain «connu». Quant aux mutations engendrées par le numérique, parce qu'elles sont plutôt liées à de la consommation (visionner des replays) qu'à de la participation (commenter les contenus), elles méritent un accompagnement créatif et positif pour développer une éducation aux médias tournée vers l'engagement. 